\title{
Frontières
}

\section{Ramener les morts parmi les vivants} Des columbariums dans les églises

\section{Jean Décarie}

Volume 15, numéro 1, automne 2002

Délires urbains, dangers de mort

URI : https://id.erudit.org/iderudit/1073910ar

DOI : https://doi.org/10.7202/1073910ar

Aller au sommaire du numéro

Éditeur(s)

Université du Québec à Montréal

ISSN

1180-3479 (imprimé)

1916-0976 (numérique)

Découvrir la revue

Citer ce document

Décarie, J. (2002). Ramener les morts parmi les vivants : des columbariums dans les églises. Frontières, 15(1), 66-68. https://doi.org/10.7202/1073910ar

Ce document est protégé par la loi sur le droit d'auteur. L'utilisation des services d'Érudit (y compris la reproduction) est assujettie à sa politique d'utilisation que vous pouvez consulter en ligne.

https://apropos.erudit.org/fr/usagers/politique-dutilisation/
Cet article est diffusé et préservé par Érudit.

Érudit est un consortium interuniversitaire sans but lucratif composé de l'Université de Montréal, l'Université Laval et l'Université du Québec à Montréal. Il a pour mission la promotion et la valorisation de la recherche. https://www.erudit.org/fr/ 


\section{Ramener les morts parmi les vivants Des columbariums dans les églises}

\section{Jean Décarie, \\ urbaniste à la retraite.}

Tout a commencé sur la montagne, au calvaire, au cimetière Notre-Dame-desNeiges. C'était dans le cadre du Plan de mise en valeur du mont Royal qui visait, on le sait, à étendre le parc à la montagne, pour le sécuriser à sa périphérie menacée par le développement institutionnel et résidentiel, mais aussi sinon surtout, pour protéger l'exceptionnel patrimoine culturel, social et architectural accumulé dans cette même couronne institutionnelle comme dans la ceinture résidentielle, toutes deux constituées par « effet de montagne » avant le parc comme tel, venu là, comme toujours les parcs, occuper les derniers terrains et les plus difficiles d'accès, les résidus de nature.

Parmi les grandes fonctions sociales investies symboliquement sur la montagne les quatre « $\mathrm{S}$ » : sacré, santé, savoir et statut social ou standing! -, la fonction funéraire a été la première dans le temps et reste la plus importante dans l'espace. Vingt-cinq ans avant le parc, dans la foulée du développement urbain industriel, des problèmes d'hygiène et des besoins d'espace qu'il entraînait, on décida de fermer les derniers cimetières villageois, sis comme il se doit près des églises paroissiales, pour les déménager hors la ville, à la campagne. Les cimetières Mount Royal et Notre-Damedes-Neiges seront ainsi inaugurés en 1852 et 1854 , selon le modèle des " cimetières ruraux » américains, souvent installés sur des hauteurs, près du ciel et loin des terres arables et rentables...

Ces « jardins célestes » seront à l'origine de la première génération des grands parcs urbains, périphériques eux aussi, de leur paysagisme pittoresque et romantique, comme plus tard du modèle de lotissement familial de la banlieue pavillonnaire ségrégationniste au tournant du siècle. Davantage, ils seront les premiers parcs avant la lettre et, comme eux, fréquentés le dimanche tant par la bourgeoisie que par le prolétariat, et tant pour le spectacle des rapports sociaux dominants, de l'ordre de la nature en exemple au désordre social avec en prime ici celui de la mort à défaut! - que pour la reproduction de la force de travail. À leur suite, les parcs seront ainsi conçus en opposition à la ville industrielle, antinomiques à la ville même, installant une dichotomie sociale et spatiale entre les nouvelles classes, entre centre et périphérie, entre culture et nature...

Le cimetière Notre-Dame-des-Neiges ajoutait au jardin anglo-saxon une dimension civique typique d'une minorité, la nôtre, voulant célébrer dans la mort les héros de sa survivance nationale. Cette fonction s'exprime par la disposition monumentale à l'entrée, mais aussi, derrière, par la superposition sur le canevas naturel, d'une trame orthogonale plus économe. Serait-ce encore cette fonction civique qui aurait incité le cimetière de la paroisse Notre-Dame à se lancer dans la construction de mausolées depuis quelques années?

On sait que le premier mausolée fut érigé en 352 av. J.-C. à Halicarnasse (Asie Mineure) par Artémise II pour son mari le roi Mausole. Une des Sept Merveilles du monde, le monument de 42 mètres de hauteur était composé de 36 colonnes ioniques supportant une pyramide où était placée la statue de Mausole. Depuis, les mausolées sont restés synonymes de grands monuments funéraires somptueux commémoratifs de personnages importants. Rien à voir avec ces hangars à cadavres somptuaires et surchauffés - moqués comme «nécro- dépôts »! -, avec leur architecture religieuse kitch, que le cimetière consent à moins de $10 \%$ de sa clientèle, à plus de 90\% italienne, extérieure à la paroisse centrale, à qui un relent de superstition d'origine inconnue (hantise du mort-vivant, régions marécageuses ?) interdit l'enterrement, la sépulture sous le niveau du sol.

Le cimetière de la paroisse Notre-Dame prétend que ces mausolées répondent simplement au besoin de s'adapter à l'évolution des rites funéraires dans une société à la fois de plus en plus multiculturelle et de moins en moins croyante. Mais d'aucuns pensent que le cimetière paroissial est devenu une entreprise commerciale, en concurrence avec «la compétition» - à $50 \%$ américaine de surcroît! - dans un marché lucratif en expansion avec le vieillissement des boomers, de façon à assurer le maintien de la basilique Notre-Dame. Cela n'est pas sans rappeler une autre braderie de ces mêmes messieurs, pour conforter leur situation financière, celle des derniers terrains du domaine des Sulpiciens, autour du Grand Séminaire.

C'est l'opposition à de tels mausolées, pour des raisons tant écologiques et économiques qu'esthétiques, qui a amené la fondation de l'Écomusée de l'Au-Delà en 1991, opposition reprise et élargie par les Amis de la Montagne, Héritage Montréal et plusieurs autres associations, au point d'obtenir de la Ville de Montréal en 1995 un moratoire sur la construction de mausolées par le cimetière Notre-Dame-desNeiges et l'obligation pour lui de produire un Plan directeur d'aménagement complet devant faire l'objet d'un Accord de développement formel. Une première version de ce plan déposée en 1997, critiquée par les organismes, avait été renvoyée par la Ville. Une version améliorée déposée en 2001 est 
en passe d'évaluation à la Ville dont la nouvelle administration a cependant annoncé, au Sommet sur le mont Royal notamment, sa préoccupation pour la préservation du patrimoine de la montagne, l'élaboration d'un nouveau Plan directeur et le retour d'instances de consultation où pareil projet devrait nécessairement être présenté.

C'est en mars 1997, lors d'un panel sur le patrimoine civique organisé par Héritage Montréal au Centre canadien d'architecture, que fut émise par nous l'idée qu'en matière de protection du patrimoine religieux, on pourrait faire d'une pierre deux coups, sauver à la fois le patrimoine du cimetière et celui des vieilles églises paroissiales en difficulté en y transférant les sépultures en mausolées ou en columbariums, ces bâtiments portant atteinte au paysage du cimetière jardin, du parc et de la montagne tout entière.

Après étude et consultation, auprès du cimetière et de ses architectes, on devait laisser tomber les enfeus en mausolées. En effet, même si plusieurs églises ont en crypte les dépouilles d'évêques et de personnages célèbres - le premier maire de Montréal, Jacques Viger, repose à l'église de NotreDame-de-Grâce - l'entreposage "industriel» des cerceuils pose des problèmes d'espace et d'entretien (ventilation, drainage, danger d'explosion) sans commune mesure avec une clientèle limitée et en diminution, ni avec la capacité d'accueil et de gestion d'une simple fabrique d'église paroissiale. Il fut donc décidé de ne retenir que la formule du columbarium, résidence des urnes cinéraires, ces petits boîtiers inertes et discrets aisément empilables sur des rayons, remisables et déplaçables, et de plus en plus populaires, l'incinération étant maintenant le choix de sépulture en pleine croissance de près de $70 \%$ de la population.

Déjà quelques églises délaissées avaient été converties en condominiums et des propositions et études étaient faites pour la conversion en columbariums, par Jean-Claude Marsan en particulier. Avec Gérard Beaudet, comme lui de la Faculté de l'aménagement de l'Université de Montréal, celui-ci avait signé en janvier 1999 une Étude sur la vocation de l'église Saint-Julien de Lachute. En mars 1999, Pierre Douillet déposait son travail de fin d'étude à l'École d'architecture de Paris-Villemin intitulé De la conversion en columbarium de l'église Saint-Louis-deFrance à Montréal. Une première visite à cette église de 1937 jamais finie mais toujours active sous le dynamique curé de
Maupeou nous a permis d'évaluer en préfaisabilité la possibilité d'implanter des ensembles de niches de part et d'autre des bas-côtés par exemple ou même dans les transepts et les jubés latéraux, sans nuire aux activités rituelles qui, avec la réduction de l'assistance, tiennent tout entières dans la nef sinon dans le chœur, ce qui pourrait permettre d'utiliser le narthex pour des cérémonies de commémoration et ajouterait au contraire, comme la présence permanente des sépultures, au caractère sacré ou spirituel parfois déficient des lieux. On estimait à environ 3000 le nombre de niches cinéraires individuelles ou familliales pouvant ainsi être logées en allées latérales et autant dans les transepts, sans compter les jubés, la sacristie et le sous-sol où pourraient être conservées les urnes moins en demande, quitte à les ressortir aux anniversaires. Cette mobilité des urnes permet enfin de concevoir éventuellement un ou des reposoirs centraux, aménagés dans des églises complètement reconverties et où seraient rassemblés les restes oubliés par le temps.

Ce type de pratique, d'aménagement et de gestion permet d'envisager une capacité de sépulture pratiquement illimitée dans une église paroissiale et dans le réseau de l'évêché, remplaçant ainsi les grands cimetières limités par l'espace ou par la valeur

L'apport ou plutôt l'appoint économique à la paroisse et au maintien de la valeur patrimoniale, architecturale, du bâtiment, n'est pas négligeable à moyen terme, mais s'ajoute surtout en effet le retour conséquent de la fonction sociale, commémorative et communautaire, des églises. Déjà celles-ci, après l'ère des bingos, multiplient en sous-sol les activités et organismes communautaires subventionnés et louent leurs clochers aux antennes de communication des réseaux de téléphonie cellulaire. La location de la nef pour les réceptions de commémorations funéraires ou simplement et éventuellement de célébrations diverses à caractère solennel sinon spirituel, pourrait ajouter à la rentabilité non seulement économique mais sociale de cette transformation du rôle des églises.

C'est dans les quartiers populaires centraux que l'on retrouve évidemment à la fois les plus anciennes, grandes et belles églises et les plus menacées par la pauvreté, par la baisse de la pratique religieuse et la baisse de la population, souvent réduite de moitié tant par le départ des nantis que par la diminution de la taille des ménages et l'augmentation des familles monoparentales. La Ville de Montréal, comme les autres prises avec le même problème, a mis en place de nombreux programmes de revitalisation de ces quartiers, portant sur le logement, l'emploi, les artères commerciales, le soutien social et familial, auxquels pourrait maintenant s'ajouter celui de la restauration des églises et de leur rôle traditionnel comme centre communautaire et non seulement paroissial, comme centre même du village devenu quartier, à côté de l'école, devant le parc, la caserne des pompiers et l'ancien hôtel de ville... Et là aussi, il pourrait $\mathrm{y}$ avoir apport financier additionnel à la préservation conséquente du patrimoine architectural religieux.

L'introduction de la fonction funéraire dans les églises paroissiales patrimoniales ne se fera évidemment pas sans études d'impact environnemental : plan d'im-

patrimoniale, et récupérant graduellement une part de l'économie funéraire accaparée par l'industrie à défaut de cimetières paroissiaux en milieu urbain. La généralisation de la crémation et l'évolution des mœurs permettent de rétablir les cimetières paroissiaux non plus derrière l'église comme autrefois ou encore dans les villages, mais directement dans l'église devenue trop grande, où la diminution ou l'évolution des pratiques fait de la place pour cette fonction on ne peut plus spirituelle et sociale, toutes confessions confondues. plantation et d'intégration architecturale
(PIIA!), évaluation avantages-coûts, analyse de gestion. C'est que le lieu est fragile, tant par la forme architecturale que par la fonction sacramentale sans compter l'indigence financière et gestionnaire. La cohabitation des sépultures avec les activités religieuses rituelles est particulièrement délicate, encore que les cendres soient moins prégnantes que les corps. À cet égard, il faudra un maximum de discrétion dans l'aménagement pour pouvoir atteindre l'objectif de contribuer au renforcement du 
caractère sacré des lieux. Il faudrait pratiquement que cela se sente sans trop se voir ! C'est pourquoi on privilégie d'abord l'utilisation des bas-côtés comme simples et sobres corridors funéraires.

Cette simplicité devrait se refléter au niveau des coûts, par l'installation de niches en étagères et par la normalisation des urnes et des affichages. Quant à la gestion, dont dépendront tant les coûts d'entretien que les bénéfices éventuels, il faudra évaluer plusieurs scénarios allant d'une simple prise en charge marginale par la fabrique paroissiale à une formule d'organisme sans but lucratif local et communautaire, à la concession à une entreprise commerciale extérieure gérant plusieurs de ces columbariums en églises. Il pourrait même s'agir des corporations des grands cimetières existants qui auraient intérêt, plutôt que d'essayer d'ériger à grands frais et esclandres de nouveaux bâtiments sans inspiration dans leurs jardins patrimoniaux, d'utiliser des bâtiments existants dédiés, magnifiques, déjà consacrés mais menacés, les églises paroissiales patrimoniales.

C'est pour répondre à ces questions qu'un projet d'étude de faisabilité fut déposé dès septembre 1999 par l'Écomusée de l'AuDelà au ministère de la Culture et des Communications du Québec dans le cadre du nouveau Programme de restauration des biens religieux du gouvernement. La demande fut transférée en février 2000 à la Fondation du patrimoine religieux, responsable de la gestion de ce programme. Létude porterait sur trois églises de Montréal choisies, en consultation avec l'abbé Turmel, vice-président de la Fondation et directeur du Comité Construction et Art sacré de l'archevêché de Montréal, comme représentatives des trois cas de figures considérés: l'église Saint-JeanBaptiste présentant le défi d'intégration maximal à cause de sa très grande valeur architecturale ; l'église Saint-Pierre-Clavert, de bonne valeur aussi, mais retenue surtout pour son expérience en gestion communautaire; et l'église Saint-Jean-de-la-Croix parce que déjà fermée et se prêtant bien au cas d'une conversion totale. Cette dernière, vendue depuis pour transformation en condos, pourrait être remplacée par l'église Saint-Louis-de-France, déjà mentionnée et étudiée.

Cette étude de faisabilité comporterait trois volets : l'analyse architecturale, le plus important, viserait à établir les critères de performance généraux tant pour l'intégration architecturale comme telle que pour la cohabitation sociale et sacramentale, aussi les coûts afférents; l'étude économique, à partir de ces coûts, par rapport à la concurrence, mais aussi en fonction du marché, de son évolution quantitative comme qualitative, la sociologie, confessions, rites, modes, formules de préarrangement, etc. ; enfin, l'analyse de la gestion, du système d'action actuel et de son évolution, des différents modèles possibles, pour déterminer le plus avantageux pour la paroisse et le positionner par rapport à la concurrence!

Après deux ans d'attente, le feu vert pour cette étude tarde à venir, hésitations et discussions entre l'État et l'Église, entre l'Église et l'Industrie, dans l'Église elle-même, entre ses cimetières, celui de la paroisse NotreDame et celui de l'archevêché. En attendant, celui-ci annonce l'abandon prochain d'une centaine d'églises à Montréal.

L'idée fait pourtant son chemin, ralliant les curés à la base, les paroissiens rencontrés et sondés, les politiciens aussi : ramener

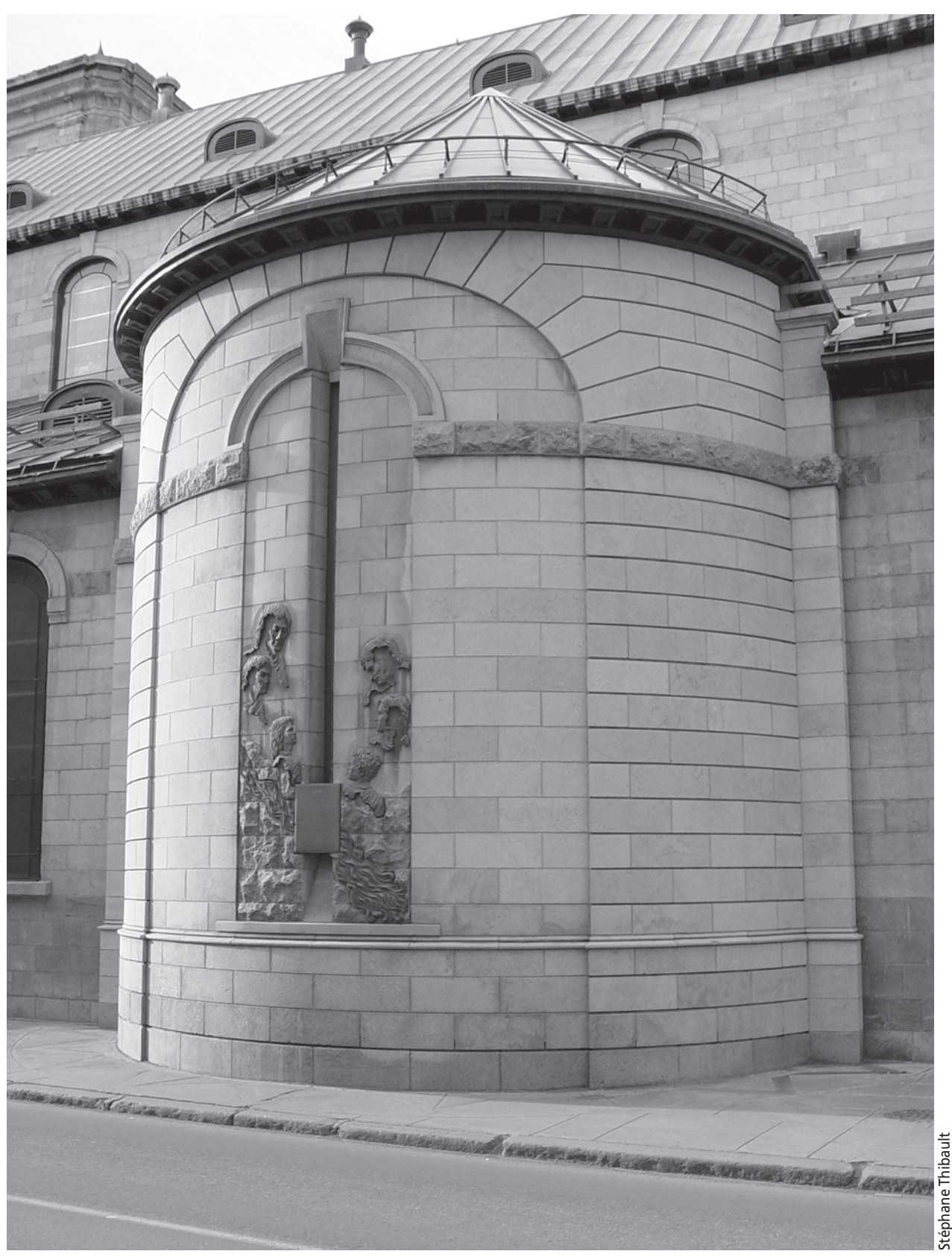

les morts parmi les vivants, faire revivre leur souvenir auprès des leurs, davantage faire en sorte que les morts eux-mêmes continuent à faire revivre l'église, la paroisse, le quartier où ils ont vécu et ainsi leur faire jouer un rôle dans le processus de production de la vie et de la ville, dans l'agir collectif, dans la vie continue, les maintenir en vie, les faire revivre presque. Pouvoir les fréquenter, les croiser, les saluer tous les jours, simplement en passant, plutôt que de les oublier, à grands frais, sur une haute colline ou dans un complexe autoroutier lointain. Pouvoir dire simplement : ils sont toujours là ! 